\title{
Book Review - Buchbesprechung - Livre nouveau
}

Mathé, G., Schwarzenberg, L. and Pouhxart, P.: Nomenclature, Methodology, and Results of Clinical Trials in Acute Leukemias; in Rentchnick, P. (ed.) Recent Results in Cancer Research, Vol. 43. Springer, Berlin, 1973. X + 168 pp.; fig. 79; tab. 64; DM 58.-/US \$ 23.80.

Diese Publikation basiert auf den Mitteilungen, die an einem internationalen Workshop am CNRS in Paris im Juni 1972 präsentiert wurden. Bei der akuten Lymphoblastenleukämie (ALL) liegt das Schwergewicht eindeutig auf der Untertei-lung in 4 zytologische Typen, welche sich mit konventioneller Färbung unterschei-den lassen. Dass diese neue Klassierung grosse diagnostische, therapeutische und prognostische Bedeutung hat, wird vor allem am Patientengut aus Villejuif doku-mentiert. Zytochemie und Elektronenmikroskopie leukämischer Zellen werden an-hand der Daten aus verschiedenen Zentren neu auf ihre Aussagekraft geprüft. Vor allem bei der Zytochemie ist auffallend, wie gross die Divergenzen der Meinungen sind. Dies kommt einerseits zustande durch methodische Unterschiede und ander-seits durch die subjektive Interpretation von Positivität bzw. Negativität. Die Ak-ten über den Wert der Zytochemie in der Diagnostik der akuten Leukämien konn-ten also auch bei diesem Workshop durchaus nicht geschlossen werden. Die thera-peutischen Trials bei ALL an verschiedenen Zentren zeigen erfreulich gute Resul-tate, und neue Wege scheinen sich anzubahnen um die Prognose noch weiter ver-bessern zu können. Bei der akuten myeloischen Leukämie (AML) sieht es trotz höherer Remissionsraten noch duster aus. Vor allem wird man pessimistisch ge-stimmt durch die kooperative Studie der europäischen Gruppe (EORTC). Im gan-zen ist das Buch für Hämatologen und Onkologen sehr stimulierend und gibt ei-nen guten Uberblick über den heutigen Stand in der Diagnostik und Therapie der akuten Leukämien.

B. Speck, Basel 